\title{
DIAGNOSIS OF THE UNRESOLVED DOMAIN TREATMENT IN MONTE CARLO TRANSPORT CALCULATIONS THROUGH THE IDENTIFICATION AND MODELLING OF CRITICALITY SAFETY EXPERIMENTS
}

\author{
N. García-Herranz, J. Rodríguez, A. Jiménez-Carrascosa and O. Cabellos \\ Universidad Politécnica de Madrid \\ José Gutiérrez Abascal, 2, 28006 Madrid, Spain \\ nuria.garcia.herranz@upm.es, jon.rodriguez.onaindia@alumnos.upm.es, \\ antonio.jcarrascosa@upm.es,oscar.cabellos@upm.es
}

\begin{abstract}
Monte Carlo neutron transport codes can be used for high-fidelity predictions of the performance of nuclear systems. However, validation against experiments is required in order to establish the credibility in the results and identify the inaccuracies due to the used calculation scheme and associated databases. The International Handbook of Evaluated Criticality Safety Benchmark Experiments (ICSBEP) contains criticality safety benchmarks derived from experiments that have been performed at various nuclear critical facilities around the world and are very valuable for validation purposes.

The main objective of this work is the identification and modelling of experimental benchmarks included at ICSBEP in support of the validation of Monte Carlo neutron transport calculations when applied to fast systems, and in particular, KENO-VI and associated AMPXformatted continuous-energy libraries from SCALE package. In such systems, the predicted k-eff values can be very sensitive to the treatment of nuclear data in the Unresolved Resonance Region (URR). Consequently, benchmarks with intermediate and fast spectra are identified and modelled with KENO-VI. Then, calculated results with and without probability tables in the URR are compared with each other in order to identify the most sensitive configurations to the URR. As a result of the proposed study, recommendations are given about the benchmarks that should be modelled and analysed to qualify the processed continuous-energy libraries before their use in Monte Carlo transport codes for practical fast reactor applications.
\end{abstract}

KEYWORDS: unresolved resonance region, probability tables, nuclear data processing, AMPXprocessed continuous-energy libraries

\section{INTRODUCTION}

The Horizon2020 European project ESFR-SMART (European Sodium Fast Reactor Safety Measures and Research Tools) [1] was launched in September 2017 with the aim of enhancing the safety of GenerationIV Sodium Fast Reactors, and in particular the commercial-size European Sodium Fast Reactor. After the French decision of dropping plans to build fast neutron reactors before the second half of this century [2], among the most useful findings of ESFR-SMART project will be those related to the calibration and validation of computational tools and methodologies to support safety assessments of innovative reactors. It becomes more important than ever to maximize the usefulness of the project by focusing on the potential drawbacks and limitations of state-of-the-art simulation tools in order to point out how to improve 
simulation-based results and how to qualify the computational approaches (computational tools, calculation schemes and associated databases) using experimental programmes.

Concerning neutronics, 3D Monte Carlo (MC) transport codes, together with continuous-energy (CE) nuclear data libraries, are the computational approaches able to conduct high-fidelity predictions of reactor core performance. However, validation against experiments is required in order to evaluate the ability of those approaches to predict the real behaviour and give credibility to the obtained results. It is recognized that nuclear data are the main source of biases and uncertainties in Monte Carlo calculations, ahead of the particular code employed. That is, nuclear data, involving both evaluated libraries and processing, are the most critical aspect impacting the quality of Monte Carlo outcomes.

Universidad Politécnica de Madrid has been involved in the past years in R\&D activities related to neutronics of Gen-IV reactors, with the final goal of contributing to improve computational methodologies for design analysis, fuel cycle performance and evaluation of safety parameters. The SCALE code system [3], developed at Oak Ridge National Laboratory (ORNL), has been used for reactor physics and sensitivity and uncertainty analysis. In particular, the SCALE's Monte Carlo transport code KENO-VI has been widely employed for neutron transport calculations.

When applying KENO-VI with AMPX-formatted Continuous Energy (CE) libraries from SCALE6.2.3 package to fast reactors, it was found that the predicted k-eff values were very sensitive to the treatment of nuclear data in the Unresolved Resonance Region (URR) [4]. It suggested that further analysis should be performed to make recommendations about how to qualify the processed CE libraries before being used for accurate Monte Carlo calculations in such fast systems.

In the URR, the cross sections have to be reconstructed from a set of statistical averages, so that codes have to deal with self-shielding [5]. Then, the cross section representation in this energy region is not as accurate as in the resolved resonance energy region and there is a significant interest in developing methodologies to improve the self-shielding treatment in the URR [6].

Any validation of the URR treatment is primarily based on computational tests. Then, in order to validate the new URR methodologies and qualify the processed nuclear data libraries for accurate Monte Carlo transport calculations in systems sensitive to the URR, the International Handbook of Evaluated Criticality Safety Benchmark Experiments (ICSBEP) can be most helpful [7]. It contains more than 5000 criticality safety benchmarks derived from experiments that have been performed at various nuclear critical facilities around the world. Those legacy data are very valuable for use in validation and qualification processes.

The main objective of this work is to identify in ICSBEP useful experimental benchmarks to diagnose the cross section treatment in the URR for the most important isotopes in fast reactors. Not only the impact on $\mathrm{k}$-eff values is analysed, but it is also worth investigating the magnitude of the effect on other parameters computed in transport calculations, such as reaction rates. No significant impact on k-eff could be found while important effects on other parameters could be identified.

The Monte Carlo neutron transport code KENO-VI and associated AMPX-formatted continuous-energy libraries from SCALE6.2.3 package (ENDF/B-VII.1-based) are employed for the analysis. Results with and without probability tables in the URR are compared with each other.

As a result of the proposed study, recommendations are given about some benchmarks that could be selected by nuclear data experts to test the validity of the developed URR methods; or could be selected by processing and end users to qualify the processed libraries before their use in Monte Carlo transport codes for practical fast reactor applications. 


\section{SELECTION OF CRITICALITY SAFETY EXPERIMENTS}

Different benchmarks from ICSBEP have been selected to assess the impact of the probability tables on important isotopes for fast reactors. Most of those benchmarks are the ones chosen by Mosteller and Little in [8]. Table 1 shows the selected uranium and plutonium cases. There are highly-enriched uranium (HEU), intermediate-enriched uranium (IEU), low-enriched uranium (LEU), mixed uranium-plutonium (MIX) and plutonium (PU) benchmarks. Most of them have a fast or intermediate spectrum, although also thermal spectrum experiments were considered for comparison purposes.

Table I. Selected benchmarks

\begin{tabular}{|c|c|c|c|c|c|}
\hline Identifier & Name & Core materials & $\begin{array}{l}\text { Reflector } \\
\text { materials }\end{array}$ & Shape & Cases \\
\hline HEU-COMP-INTER-003 & $\mathrm{UH}_{3}$ & $\begin{array}{l}\text { HEU (93 wt.\%) } \\
\text { Hydride }\end{array}$ & $\begin{array}{l}\text { Depleted U, } \\
\text { Iron, } \\
\text { Beryllium }\end{array}$ & Cylindrical & $\begin{array}{c}-001,-002,-003, \\
-004,-005,-006, \\
-007\end{array}$ \\
\hline HEU-COMP-INTER-004 & HISS/HUG & $\begin{array}{l}\text { HEU (92 wt.\%), } \\
\text { Graphite, Boron }\end{array}$ & - & Infinite & -001 \\
\hline HEU-MET-FAST-001 & GODIVA & HEU (93 wt.\%) & None & Spherical & -001 \\
\hline HEU-MET-INTER-006 & ZEUS & $\begin{array}{c}\text { HEU (93 wt.\%), } \\
\text { Graphite }\end{array}$ & Copper & Cylindrical & $\begin{array}{c}-001,-002,-003, \\
-004\end{array}$ \\
\hline HEU-SOL-THERM-013 & ORNL-4 & $\begin{array}{l}\text { HEU (93 wt.\%) } \\
\text { Nitrate Solution }\end{array}$ & None & Spherical & $\begin{array}{c}-001,-002,-003, \\
-004\end{array}$ \\
\hline IEU-COMP-FAST-004 & ZPR-III-12 & $\begin{array}{c}\text { IEU (21 wt.\%), } \\
\text { Graphite }\end{array}$ & $\begin{array}{c}\text { Depleted U, } \\
\text { Steel }\end{array}$ & Cylindrical & - \\
\hline IEU-MET-FAST-004 & $\begin{array}{c}\text { Graphite- } \\
\text { Reflected IEU } \\
\text { Sphere }\end{array}$ & IEU (36 wt.\%) & Graphite & Spherical & DET and SIM \\
\hline IEU-MET-FAST-007 & BIG TEN & IEU (10 wt.\%) & Depleted U & Cylindrical & DET and SIM \\
\hline LEU-SOL-THERM-001 & SHEBA-II & $\begin{array}{c}\text { LEU (5 wt.\%) } \\
\text { Uranyl Fluoride, } \\
\text { Water }\end{array}$ & None & Cylindrical & -001 \\
\hline MIX-COMP-FAST-001 & $\begin{array}{c}\text { ZPR-6 } \\
\text { Assembly } 7\end{array}$ & $\begin{array}{l}\text { Mixed (Pu,U)- } \\
\text { Oxide, Sodium }\end{array}$ & Depleted U & Cylindrical & \\
\hline MIX-COMP-FAST-006 & ZPPR-2 & $\begin{array}{l}\text { Mixed }(\mathrm{Pu}, \mathrm{U})- \\
\text { Oxide, Sodium }\end{array}$ & $\begin{array}{c}\text { Depleted U, } \\
\text { Sodium and } \\
\text { Steel }\end{array}$ & Cylindrical & \\
\hline PU-COMP-INTER-001 & HISS/HPG & $\begin{array}{c}\mathrm{Pu}(5.3 \text { at. } \% 240 \mathrm{Pu}), \\
\text { Graphite, Boron }\end{array}$ & - & Infinite & -001 \\
\hline PU-MET-FAST-001 & JEZEBEL & $\mathrm{Pu}(4.5$ at. $\% 240 \mathrm{Pu})$ & None & Spherical & \\
\hline PU-MET-FAST-002 & $\begin{array}{l}\text { JEZEBEL- } \\
240 \\
\end{array}$ & $\begin{array}{c}\mathrm{Pu}(20.1 \text { at.\% } \\
240 \mathrm{Pu})\end{array}$ & None & Spherical & -001 \\
\hline PU-SOL-THERM-021 & PNL-2 & $\begin{array}{c}\mathrm{Pu}(4.5 \text { at. } \% 240 \mathrm{Pu}) \\
\text { Nitrate, Water }\end{array}$ & None & Spherical & $\begin{array}{c}-001,-002,-003, \\
-004,-005,-006, \\
-007,-008,-009, \\
-010\end{array}$ \\
\hline
\end{tabular}

The benchmarks were modelled for KENO-VI neutron transport Monte Carlo code in continuous energy mode, starting from the inputs available in the handbook for MMK-KENO code, a multi-group Monte Carlo code based on KENO-V.a. Then, KENO-VI results with and without probability tables (PT) in the URR were computed (PT were disabled for all isotopes at once). Each calculation employed a maximum of 
10,000 generations of 10,000 neutrons each, and 100 skipped generations, in order to get a statistical deviation around $10 \mathrm{pcm}$.

A Python script was developed to compare not only k-eff values but also microscopic cross-sections, reaction rates and flux spectrum in the $175 \mathrm{~g}$-VITAMIN-J structure, as well as in the URR energy ranges of the main isotopes. For each experiment, an analysis of the sensitivities of k-eff available in DICE [9], an user-friendly tool developed by the Nuclear Energy Agency, was performed in order to point out the cross sections with the highest impact on reactivity and focus the analysis on those reactions.

\section{RESULTS WITH AND WITHOUT PROBABILITY TABLES}

\subsection{Comparison of k-eff values}

Table II shows the k-eff values with and without PT in the URR along with their statistical standard deviations. Among the HEU benchmarks, it can be seen that the $\mathrm{UH}_{3}$ benchmark is the most sensitive to the PT treatment, in particular the first case. Concerning IEU benchmarks, two cases exhibited significant changes in reactivity when PT were not considered, ZPR-III-12 and BIG TEN, both detailed and simplified cases. The mixed U-Pu benchmarks analysed showed a substantial sensitivity to the PT. The only Pu benchmark with a significant difference is the HISS/HPG, which has intermediate spectra, while the rest of cases are not affected. Finally, as expected, the PT treatment does not impact at all the benchmarks with thermal spectrum.

Table II. Effect of PT on k-eff

\begin{tabular}{|l|c|c|c|r|}
\hline \multirow{4}{*}{ Identifier } & Case & $\begin{array}{c}\text { Calculated } \\
\text { k-eff with PT }\end{array}$ & $\begin{array}{c}\text { Calculated } \\
\text { k-eff without PT }\end{array}$ & $\begin{array}{c}\Delta \text { keff } \\
\text { (pcm })\end{array}$ \\
\hline \multirow{5}{*}{ HEU-COMP-INTER-003 } & -001 & $1.00705 \pm 0.0001$ & $1.00841 \pm 0.0001$ & $-135 \pm 14$ \\
\cline { 2 - 5 } & -002 & $1.02931 \pm 0.0001$ & $1.03029 \pm 0.0001$ & $-99 \pm 14$ \\
\cline { 2 - 5 } & -003 & $0.99579 \pm 0.0001$ & $0.99673 \pm 0.0001$ & $-95 \pm 14$ \\
\cline { 2 - 5 } & -004 & $1.00325 \pm 0.0001$ & $1.00424 \pm 0.0001$ & $-99 \pm 14$ \\
\cline { 2 - 5 } & -005 & $0.99751 \pm 0.0001$ & $0.99775 \pm 0.0001$ & $-24 \pm 14$ \\
\cline { 2 - 5 } & -006 & $0.99848 \pm 0.0001$ & $0.99935 \pm 0.0001$ & $-87 \pm 14$ \\
\cline { 2 - 5 } & -007 & $0.99433 \pm 0.0001$ & $0.99518 \pm 0.0001$ & $-85 \pm 14$ \\
\hline HEU-COMP-INTER-004 & -001 & $1.01192 \pm 0.0001$ & $1.01188 \pm 0.0001$ & $4 \pm 14$ \\
\hline HEU-MET-FAST-001 & -001 & $0.99982 \pm 0.0001$ & $1.00002 \pm 0.0001$ & $-20 \pm 14$ \\
\hline \multirow{5}{*}{ HEU-MET-INTER-006 } & -001 & $0.99347 \pm 0.0001$ & $0.99417 \pm 0.0001$ & $-70 \pm 14$ \\
\cline { 2 - 6 } & -002 & $0.99992 \pm 0.0001$ & $1.00040 \pm 0.0001$ & $-48 \pm 14$ \\
\cline { 2 - 5 } & -003 & $1.00085 \pm 0.0001$ & $1.00168 \pm 0.0001$ & $-82 \pm 14$ \\
\cline { 2 - 5 } & -004 & $1.00561 \pm 0.0001$ & $1.00641 \pm 0.0001$ & $-80 \pm 14$ \\
\hline \multirow{5}{*}{ HEU-SOL-THERM-013 } & -001 & $0.99837 \pm 0.0001$ & $0.99852 \pm 0.0001$ & $-14 \pm 14$ \\
\cline { 2 - 5 } & -002 & $0.99770 \pm 0.0001$ & $0.99771 \pm 0.0001$ & $-1 \pm 14$ \\
\cline { 2 - 5 } & -003 & $0.99411 \pm 0.0001$ & $0.99422 \pm 0.0001$ & $-11 \pm 14$ \\
\cline { 2 - 5 } & -004 & $0.99580 \pm 0.0001$ & $0.99582 \pm 0.0001$ & $-2 \pm 14$ \\
\hline
\end{tabular}




\begin{tabular}{|l|c|r|r|r|}
\hline IEU-COMP-FAST-004 & -001 & $1.00039 \pm 0.0001$ & $0.99820 \pm 0.0001$ & $219 \pm 14$ \\
\hline \multirow{3}{*}{ IEU-MET-FAST-004 } & DET & $1.00770 \pm 0.0001$ & $1.00789 \pm 0.0001$ & $18 \pm 14$ \\
\cline { 2 - 5 } & SIM & $1.00735 \pm 0.0001$ & $1.00786 \pm 0.0001$ & $51 \pm 14$ \\
\hline \multirow{3}{*}{ IEU-MET-FAST-007 } & DET & $1.00554 \pm 0.0001$ & $1.00083 \pm 0.0001$ & $471 \pm 14$ \\
\cline { 2 - 5 } & SIM & $1.00536 \pm 0.0001$ & $1.00072 \pm 0.0001$ & $464 \pm 14$ \\
\hline LEU-SOL-THERM-001 & -001 & $1.01145 \pm 0.0001$ & $1.01123 \pm 0.0001$ & $-22 \pm 14$ \\
\hline MIX-COMP-FAST-001 & & $0.99074 \pm 0.0003$ & $0.98506 \pm 0.00003$ & $569 \pm 32$ \\
\hline MIX-COMP-FAST-006 & & $0.99490 \pm 0.0001$ & $0.98989 \pm 0.00003$ & $501 \pm 8$ \\
\hline PU-COMP-INTER-001 & -001 & $1.01297 \pm 0.0001$ & $1.01174 \pm 0.0001$ & $123 \pm 14$ \\
\hline PU-MET-FAST-001 & -001 & $1.00109 \pm 0.0001$ & $1.00096 \pm 0.0001$ & $13 \pm 14$ \\
\hline PU-MET-FAST-002 & -001 & $1.00025 \pm 0.0001$ & $1.00036 \pm 0.0001$ & $-11 \pm 14$ \\
\hline \multirow{5}{*}{ PU-SOL-THERM-021 } & -001 & $1.00489 \pm 0.0001$ & $1.00500 \pm 0.0001$ & $-11 \pm 14$ \\
\cline { 2 - 5 } & -002 & $1.00498 \pm 0.0001$ & $1.00520 \pm 0.0001$ & $-22 \pm 14$ \\
\cline { 2 - 5 } & -003 & $1.00449 \pm 0.0001$ & $1.00459 \pm 0.0001$ & $-10 \pm 14$ \\
\cline { 2 - 5 } & -004 & $0.36992 \pm 0.0001$ & $0.36997 \pm 0.0001$ & $-6 \pm 14$ \\
\cline { 2 - 5 } & -005 & $1.00463 \pm 0.0001$ & $1.00464 \pm 0.0001$ & $0 \pm 14$ \\
\cline { 2 - 5 } & -006 & $1.00695 \pm 0.0001$ & $1.00709 \pm 0.0001$ & $-14 \pm 14$ \\
\cline { 2 - 5 } & -007 & $1.00617 \pm 0.0001$ & $1.00623 \pm 0.0001$ & $-5 \pm 14$ \\
\cline { 2 - 5 } & -008 & $1.00356 \pm 0.0001$ & $1.00345 \pm 0.0001$ & $11 \pm 14$ \\
\cline { 2 - 5 } & -009 & $1.00661 \pm 0.0001$ & $1.00651 \pm 0.0001$ & $10 \pm 14$ \\
\cline { 2 - 5 } & -010 & $1.00638 \pm 0.0001$ & $1.00629 \pm 0.0001$ & $10 \pm 14$ \\
\hline
\end{tabular}

\subsection{Comparison of reaction rates}

A comparison of the reaction rates over the whole system computed with and without PT in $175 \mathrm{~g}$ was performed. Relative deviations for the main $\mathrm{U}$ and $\mathrm{Pu}$ isotopes were also calculated in 3 energy groups: below the corresponding URR, in the URR and above the URR. In the employed ENDF/B-VII.1 library, the URR for U-235 extends from $2.25 \mathrm{keV}$ to $25 \mathrm{keV}$; the URR for U-238 extends from $20 \mathrm{keV}$ to $149 \mathrm{keV}$, while ranges for Pu-239, Pu-240 and Pu-241 are (2.5 keV-30 keV), $(5.7 \mathrm{keV}-40 \mathrm{keV})$ and $(0.3 \mathrm{keV}-40.2$ $\mathrm{keV}$ ) respectively.

The relative differences in reaction rates in the URR for HEU benchmarks are shown in Figure 1, along with the fraction of the interactions over the core that take place within the URR.

It can be observed that the PT treatment does not change significantly the U-235 reaction rates for any of the analysed cases, being relative differences in the corresponding URR lower than about $1 \%$.

However, differences in U-238 reaction rates up to $\sim 8 \%$ in the URR were found for some cases of the $\mathrm{UH}_{3}$ benchmark (HEU-COMP-INTER-003-), corresponding to the cases where reactivity impact of PT was around $100 \mathrm{pcm}$ or higher. A detailed analysis showed that the presence of a reflector of depleted uranium in the $\mathrm{UH}_{3}$ benchmark is the only responsible of the differences. While the impact of URR on case -001 is $135 \mathrm{pcm}$ (inner and outer reflector of depleted uranium), in case -005 it is negligible (inner reflector of Be). The intermediate spectrum in the depleted uranium reflector in case -001 (represented in Figure 2.a) leads to significant deviations in the U-238 capture cross-section in this material (Figure 2.b). 


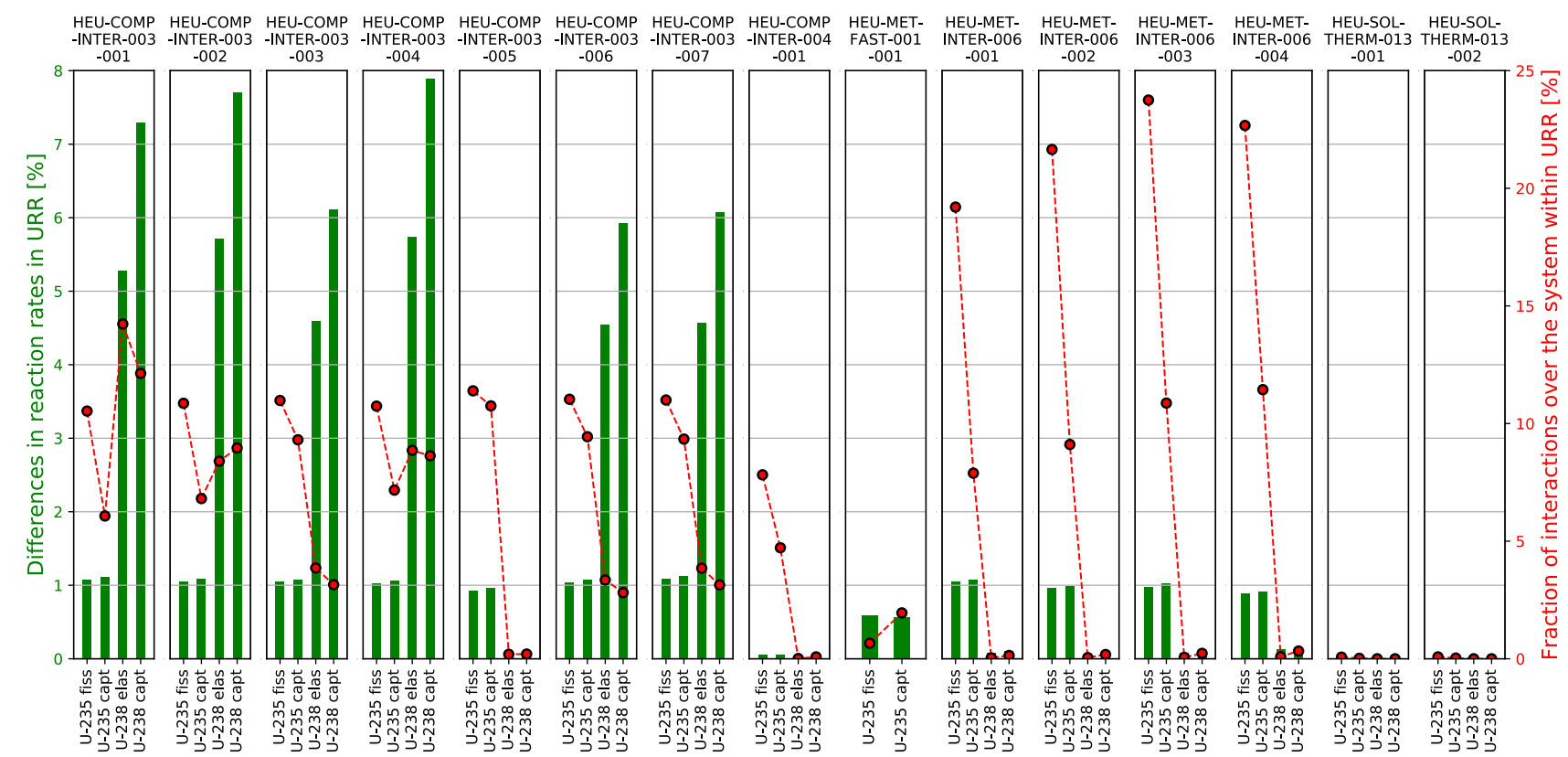

Figure 1. Differences in reaction rates in the URR with and without PT and fraction of interactions within the URR for HEU benchmarks. Relative values computed as $\left(\mathbf{r r}_{\mathrm{noPT}}-\mathbf{r r}_{\mathrm{PT}}\right) / \mathbf{r r}_{\mathrm{PT}}$

The rest of HEU benchmarks are not significantly affected by the PT treatment since a negligible fraction of U-238 reactions are within the URR, due to a small amount of U-238 present in the system. In summary, none of the analysed HEU benchmarks are useful to assess the PT treatment in U-235, but $\mathrm{UH}_{3}$ cases with a depleted uranium reflector are very convenient to analyse the PT treatment of U-238 due to significant differences in captures, even if the impact on k-eff is not huge and under this criteria would not be selected.
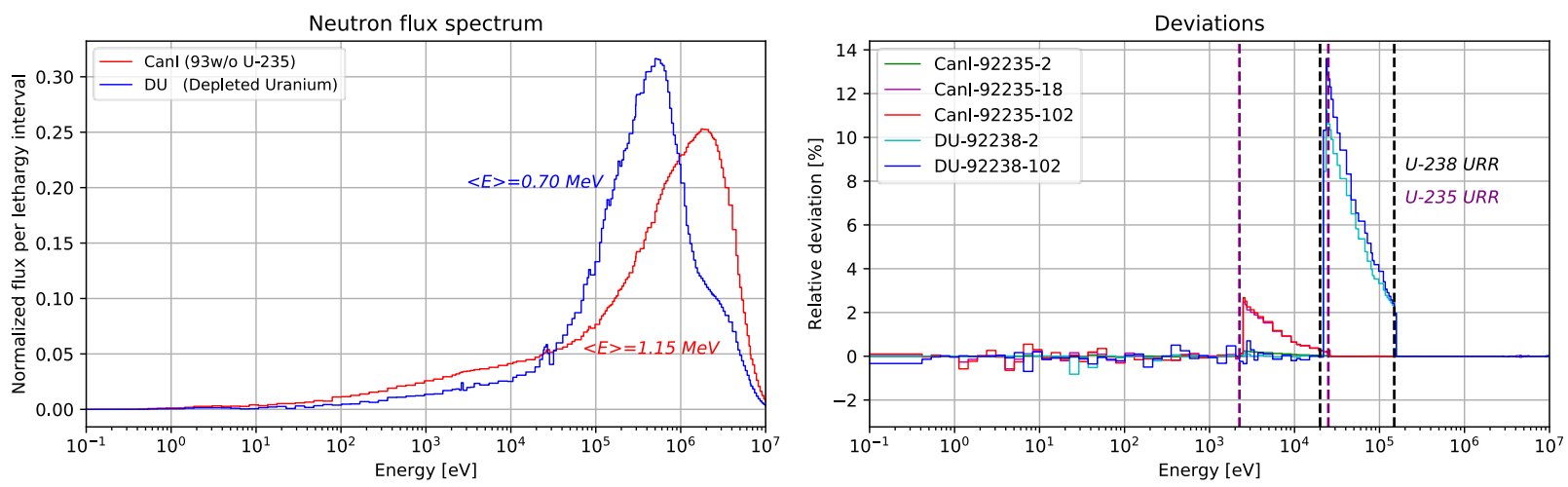

Figure 2. (a) Neutron flux spectrum at Can I with 93w/o U-235 and at the depleted uranium reflector of HEU-COMP-INTER-003-001 benchmark. (b) Relative differences in cross-sections with and without PT in the URR in those materials

Figure 3 shows the relative differences in reaction rates in the URR, together with the fraction of reactions over the system in the URR, for IEU and LEU benchmarks. Maximum differences of $4 \%$ in the URR of U238 capture and elastic reaction rates appear in IEU cases, but the global impact will be significant only for those cases where a large fraction of interactions over the core is within the URR. Such is the case for ZPRIII-12 (IEU-COMP-FAST-004), where $46 \%$ of the total captures occur in U-238 within its URR, or for BIG TEN (IEU-MET-FAST-007), with 44\% of total captures in the U-238 URR. A comparison of the results for detailed and simplified cases of BIG TEN showed that they are equivalent from a spectral point of view, 
with nearly the same fractions of interactions in the URR (the same can be said for ZPR-III-12); consequently, the impact of PT treatment is very similar for detailed and simplified cases. As in HEU benchmarks, in IEU cases the impact of the PT is also due to U-238.
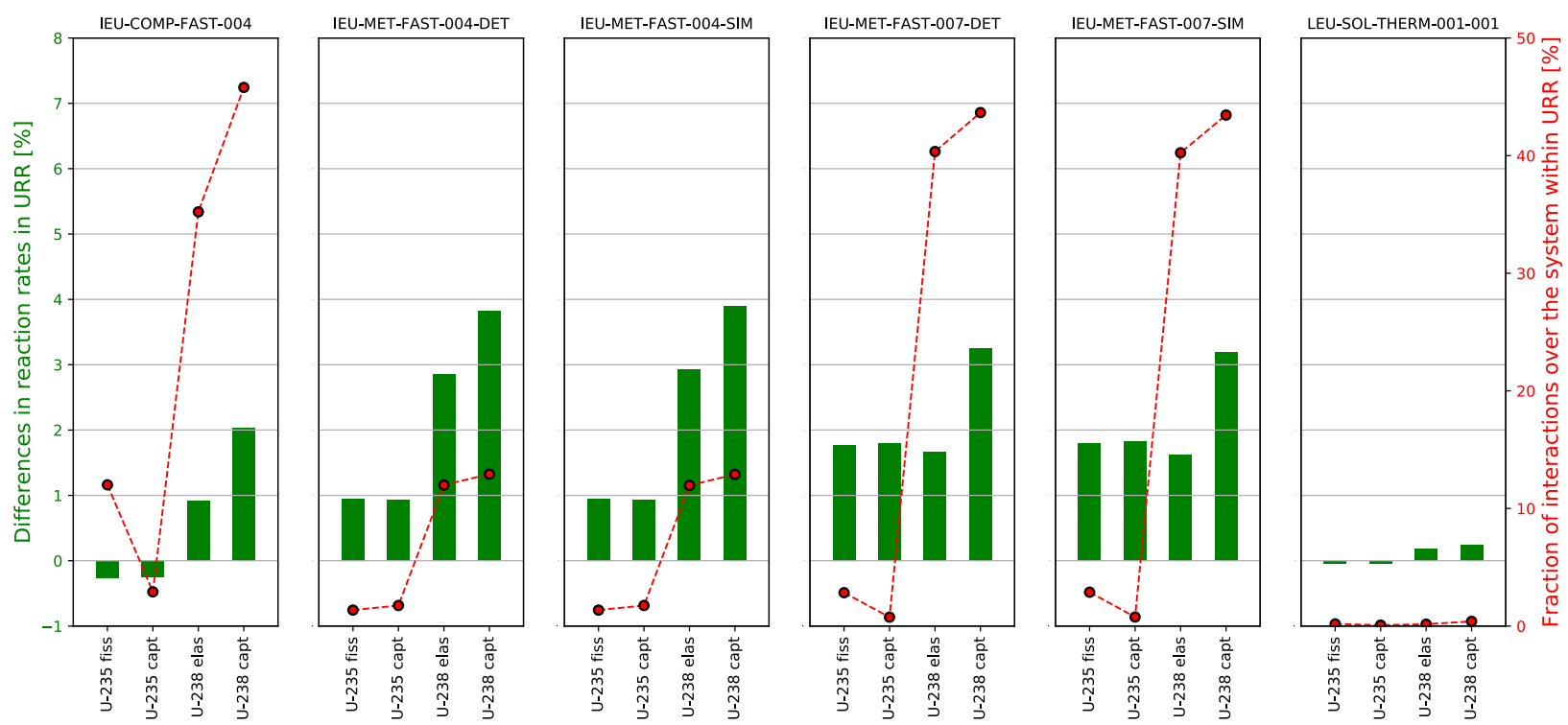

Figure 3. Differences in reaction rates in the URR with and without PT and fraction of interactions within the URR for IEU and LEU benchmarks. Relative values computed as $\left(\mathbf{r r}_{\mathrm{noPT}}-\mathbf{r r}_{\mathrm{PT}}\right) / \mathbf{r r}_{\mathrm{PT}}$

Results for the mixed uranium-plutonium and plutonium benchmarks are in Figure 4. Both MIX benchmarks are useful to analyse the PT treatment of U-238 and Pu-239, since there are differences in their reaction rates and a significant fraction of those reactions are within the corresponding URR. The most sensitive Pu benchmark is PU-COMP-INTER-001, although MET-FAST benchmarks exhibit also a nonnegligible fraction of Pu-239 captures in the URR together with relevant differences in reaction rates. In the Pu benchmarks with thermal spectrum, the fraction of interactions in the URR is insignificant.
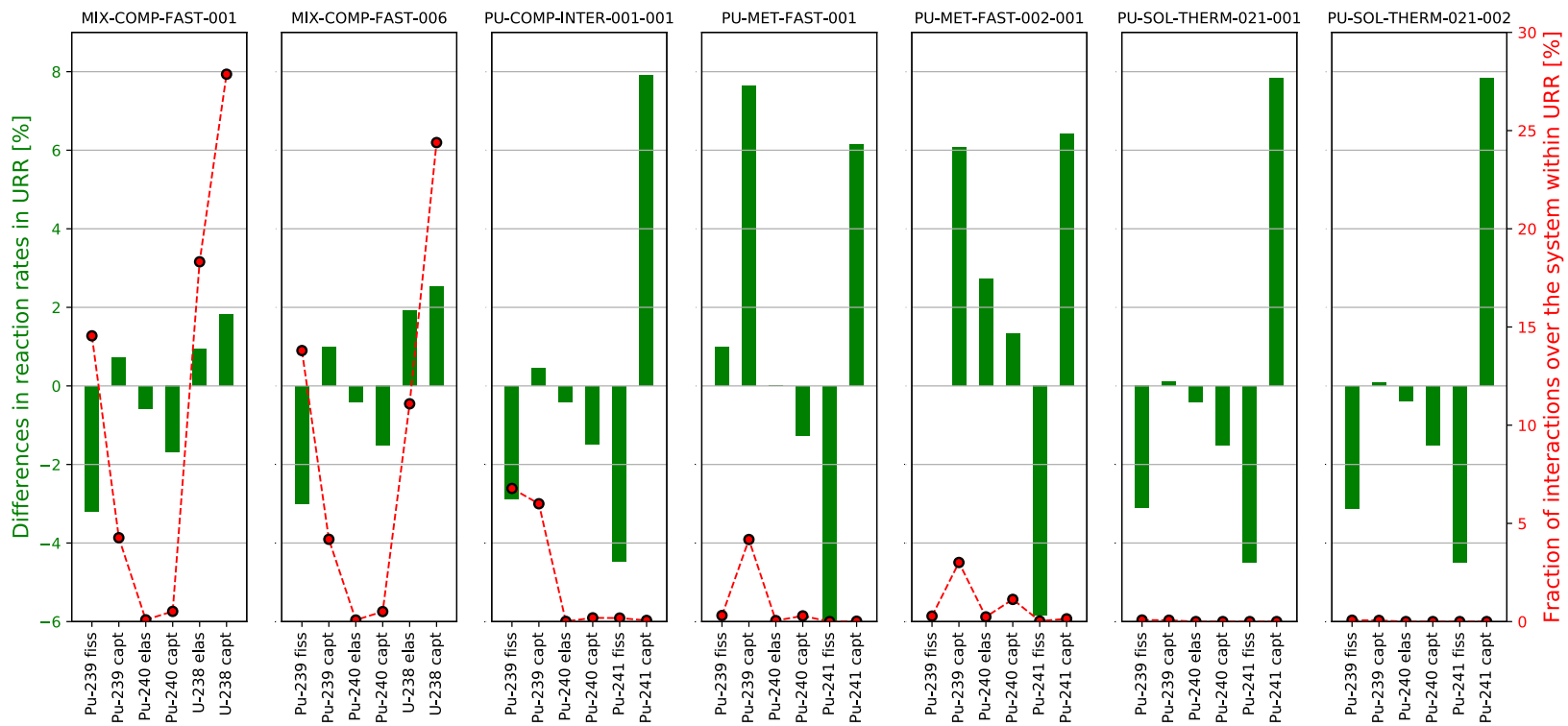

Figure 4. Differences in reaction rates in the URR with and without PT and fraction of interactions within the URR for MIX and Pu benchmarks. Relative values computed as $\left(\mathbf{r r}_{\text {noPT }}-\mathbf{r r}_{\mathrm{PT}}\right) / \mathbf{r r}_{\mathrm{PT}}$ 


\section{CONCLUSIONS}

Typically, in order to assess the impact of the probability table treatment, comparisons of reactivity with and without PT is carried out. In this study, a comparison of reaction rates has been also performed. The PT treatment impacts reaction rates of the main uranium and plutonium isotopes. However, based only on relative differences it is not possible to identify the most sensitive benchmarks to the PT treatment; the amount of isotopes present in the system as well as the URR spectrum overlap (or similarly, the fraction of interactions over the system within the unresolved resonance range of each isotope) determine the magnitude of the impact.

Results for HEU experiments indicate that HEU-COMP-INTER-003 benchmark, cases -001, -002, -003, 004 are useful to assess the PT impact of U-238 due to the large amount of U-238 in the depleted uranium reflector. The PT treatment of U-238 also produces significant differences in two IEU benchmarks, IEUCOMP-FAST-004 and IEU-MET-FAST-007, as well as in two mixed-uranium plutonium benchmarks. Only one of the analysed $\mathrm{Pu}$ benchmarks showed sensitivity to the PT treatment of Pu-239, being the effect on the rest negligible.

Only few benchmarks from ICSBEP have been analysed. Future work should focus on identifying all the potential benchmarks sensitive to the URR for the different isotopes of interest in fast reactors and analyze the most affected parameters.

\section{ACKNOWLEDGMENTS}

The research conducted was made possible through funding from the Euratom research and training programme 2014-2018 under grant agreement No 754501 (ESFR-SMART project).

\section{REFERENCES}

1. K. Mikityuk et al., "ESFR-SMART: new Horizon-2020 project om SFR safety," Proceedings of the International Conference on Fast Reactors and Related Fuel Cycles: Next Generation Nuclear Systems for Sustainable, Development (FR17), Yekaterinburg, Russia, June 26-29, 2017 (2018).

2. N. Wakin, Le Monde, Nucléaire: la France abandonne la quatrième generation de réacteurs, 29 août 2019.

3. "SCALE code, SCALE 6.2.3" https://www.oecd-nea.org/tools/abstract/detail/ccc-0834/ (2018).

4. A. Jiménez-Carrascosa, E. Fridman, N. García-Herranz, F. Alvarez-Velarde, P. Romojaro, F. Bostelmann, "About the impact of the Unresolved Resonance Region in Monte Carlo simulations of Sodium Fast Reactors", Proceedings of the International Congress on Advances in Nuclear Power Plants (ICAPP 2019), France, Juan-les-pins, May 12-15 (2019).

5. Cullen D.E., "The Importance of Resonance Self-Shielding”, INDC Int. Nucl. Data Committee, INDC(NDS)-0778, IAEA (2019).

6. A. Holcomb, L. Leal, F. Rahnema, D. Wiarda, "A new method for generating probability tables in the Unresolved Resonance Region”, Nucl. Sci. and Eng., DOI: 10.1080/00295639.2016.1273632 (2016).

7. International Criticality Safety Benchmark Evaluation Project (ICSBEP) // https://www.oecdnea.org/science/wpncs/icsbep/handbook.html (2018).

8. R.D. Mosteller, R. C. Little, "Impact of MCNP Unresolved Resonance Probability-Table Treatment on Uranium and Plutonium Benchmarks", LA-UR-99-2300, $6^{\text {th }}$ Int. Conf. on Nuclear Criticality Safety, France, September 20-24 (1999).

9. I. Hill et al., "DICE 2013: New capabilities and data", Proceedings of the International Conference on Physics of Reactors (PHYSOR2014), Kyoto, Japan, September 28 - October 3 (2014). 